\title{
Optimization of Culture Conditions of Porcellio dilatatus (Crustacea: Isopoda) for Laboratory Test Development
}

\author{
Isabel Caseiro, ${ }^{*}$ S. Santos, $\dagger$ J. P. Sousa, ${ }^{* 1}$ A. J. A. Nogueira, ${ }^{*}$ and A. M. V. M. Soares* + \\ *Instituto Ambiente e Vida, Departamento de Zoologia, Universidade de Coimbra, 3004-517 Coimbra, Portugal; †Escola Superior Agrária de Bragança, \\ Instituto Politécnico de Bragança, Bragança, Portugal; and $\ddagger$ Departamento de Biologia, Universidade de Aveiro, Aveiro, Portugal
}

Received December 21, 1999

This paper describes the experimental results for optimizing isopod culture conditions for terrestrial ecotoxicity testing. The influence of animal density and food quality on growth and reproduction of Porcellio dilatatus was investigated. Results indicate that density influences isopod performance in a significant way, with low-density cultures having a higher growth rate and better reproductive output than medium- or high-density cultures. Alder leaves, as a soft nitrogen-rich species, were found to be the best-quality diet; when compared with two other food mixtures, alder leaves induced the best results, particularly in terms of breeding success. Guidance regarding culture conditions of isopod species, especially in terms of culture density and diet, is given. (c) 2000 Academic Press

Key Words: isopods; Porcellio dilatatus; density; food quality; growth; reproduction.

\section{INTRODUCTION}

Terrestrial isopods are good representatives of the soil macrodecomposer group. Their saprophytic activity contributes in a significant way to litter fragmentation, increasing the attack area for decomposers, constituting an important step for the decomposition process. The activity of these organisms increases the recycling of essential nutrients, which have a great importance for the maintenance of soil fertility (Hopkin and Martin, 1982; Van Straalen and Verweij, 1991).

These ecological characteristics associated with the facilitated capture in the field and maintenance in laboratory cultures make terrestrial isopods good candidates for test organisms, for both purely ecological and ecotoxicological studies (Donker, 1992; Drobne, 1997). In fact, the number of studies with these organisms has been increasing in the past few years (Donker et al., 1993; Leon and Van Gestel, 1994) and isopods are even considered as potential test organisms

\footnotetext{
${ }^{1}$ To whom correspondence should be addressed. Fax: + 351239826798 E-mail: jps@zoo.uc.pt.
}

in a tiered approach for evaluating the effects of toxic substances in terrestrial systems (Römbke et al., 1996). Most studies use animals coming directly from the field or maintained in the laboratory as temporary cultures for that specific purpose. These procedures, however, do not fit the needs of regular use of these organisms for the evaluation of several anthropogenic actions in the terrestrial environment that require the maintenance of laboratory cultures under controlled conditions. By using cultured individuals the necessary number and type of animals (sex, age class) can be obtained in the best physiological conditions. Thereby it is necessary to develop several procedures to maintain appropriate laboratory cultures of isopods.

Among others, climatic conditions, animal density, and food quality and availability can be considered as the most important factors in regulating population dynamics of isopod species (Merriam, 1971; Rushton and Hassall, 1987; Hassall and Dangerfield, 1997). Several studies indicate that density affects the size of isopod populations (Hassall, 1983; Van Wensem, 1989) and that interactions between isopods may reduce their growth, survivorship, and reproduction (Ganter, 1984). Regarding diet, the studies of Merriam (1971) and Rushton and Hassall (1983b) indicated that food quality can cause important changes in individual and population parameters. These authors demonstrated that food quality could significantly change growth rate in Armadillidium vulgare, directly affecting the time of the first reproduction, a key factor in controlling population growth. Hassall and Rushton (1984) demonstrated that isopods can be very selective in regulating their diet, exhibiting a marked preference for certain types of leaf litter. Freshly fallen leaves from dicotyledonous plants provide the best-quality food for isopods, in contrast to leaves from monocotyledonous plants which are of poor quality (Rushton and Hassall, 1983a).

The aim of the present study was to optimize laboratory culture conditions, evaluating the effects of density (number of animals per culture box) and food quality on growth and reproductive output of the terrestrial woodlouse Porcellio dilatatus. The choice of $P$. dilatatus was based on the 
representativity of this species in Southern Europe. The genus Porcellio has, essentially, an occidental distribution (Vandel, 1962) and P. dilatatus is particularly well represented in the Atlantic Islands (Madeira, Azores, and Canary Islands), Iberian Peninsula, and North African countries (Morocco, Algeria, and Tunisia). Its numbers in Britain and Ireland (and perhaps in other Central European countries) may be underestimated due to confusion with $P$. scaber (Hopkin, 1991). According to this author, P. dilatatus is very common in a variety of synanthropic habitats (e.g., farms) and it is easy to maintain in laboratory cultures, making it a suitable candidate for use in ecotoxicological studies.

\section{MATERIALS AND METHODS}

\section{Test Organism and Culture Procedure}

The isopods used in these experiments ( $P$. dilatatus) were obtained from laboratory cultures, composed of animals collected from a dunar system in central Portugal. The animals were kept at $22^{\circ} \mathrm{C}$ in plastic boxes with a layer of sand (approximately $2 \mathrm{~cm}$ ) and fed alder leaves (Alnus glutinosa). Some holes were made on the box cover to ensure ventilation, and periodic spraying with distilled water was sufficient to guarantee adequate moisture conditions. To study the effect of density and food quality on isopod performance two experiments were made.

\section{Density Experiment (Experiment 1)}

Three different densities were established: 6, 9, and 12 animals per culture box. For each density, 10 replicates were used with animals with an initial average weight of $6.1 \mathrm{mg}$. Isopods were kept at $22^{\circ} \mathrm{C}$ in plastic boxes $\left(116 \mathrm{~cm}^{2}\right)$ with a layer of sand under a constant photoperiod $(16 \mathrm{~h} \mathrm{light:} 8 \mathrm{~h}$ dark). Alder leaves (A. glutinosa) were previously dried in the oven for $48 \mathrm{~h}$ at $60^{\circ} \mathrm{C}$, remoistened, and offered ad libidum to isopods.

Isopods were weighed weekly for 10 weeks. During this time, food and distilled water were added on a regular basis. The water supply was made with a pipet directly on the substrate to maintain humidity without excessive development of fungi. To maintain the initial density, isopods found dead were replaced by others of identical weight and size. This experiment involved the study of the first and second generations; juveniles from the first generation were collected and transferred to identical boxes with the same conditions (abiotic factors, food, and density) for study of the second generation. The parameters measured were growth and reproductive performance (number of pregnant females and number of juveniles/female).

\section{Food Quality Experiment (Experiment 2)}

For this experiment, three diets were chosen: alder leaves, mixture 1 , and mixture 2 . The composition of mixture 1 was
TABLE 1

Composition of the Three Food Types Tested (Average Values \pm SD)

\begin{tabular}{lccc}
\hline Parameter & Alder & Mixture 1 & Mixture 2 \\
\hline Nitrogen (\% N) & $3.72 \pm 0.07$ & $3.03 \pm 0.02$ & $2.40 \pm 0.01$ \\
Phosphoros (\% P) & $0.18 \pm 0.01$ & $0.38 \pm 0.01$ & $0.45 \pm 0.1$ \\
Potassium (\% K) & $1.05 \pm 0.02$ & $1.24 \pm 0.08$ & $1.82 \pm 0.02$ \\
Sulfur (\% S) & $0.18 \pm 0.1$ & $0.17 \pm 0.01$ & $0.15 \pm 0.01$ \\
Calcium (\% Ca) & $0.85 \pm 0.01$ & $4.79 \pm 0.08$ & $6.77 \pm 0.1$ \\
Iron (ppm Fe) & $434.67 \pm 11.02$ & $298 \pm 1.73$ & $140 \pm 8.54$ \\
\hline
\end{tabular}

Note. Values are expressed in terms of dry weight $\left(105^{\circ} \mathrm{C}\right)$.

$47 \%$ alder leaves, $15 \%$ potato, $15 \%$ carrot, $15 \%$ Purina, and $8 \% \mathrm{CaCO}_{3}$. Mixture 2 contained $72 \%$ carrot, $14 \%$ of alder leaves, and $14 \% \mathrm{CaCO}_{3}$. All these substances, except $\mathrm{CaCO}_{3}$, were previously dried in the oven for $48 \mathrm{~h}$ and then reduced to powder with a kitchen blender.

Chemical characterization of the different diets is represented in Table 1. The choice of these components was based on their role in the development of isopods, the food preferences of these animals, and also the ease with which they could be obtained and manipulated. Carrots and potatoes are an important source of carbohydrates, leading to an increase in growth rate in isopods (Carefoot, 1984; Warburg, 1995), and are frequently used in laboratory cultures by several authors (Brody and Lawlor, 1984; Wieser and Oberhauser, 1984; Hopkin, 1991; Bayley, 1995). Merriam (1971) suggested Purina Rabbit Chow as a high-quality diet, with a balanced composition in proteins, fat, and fiber, inducing good performance in isopods. Finally, calcium carbonate was chosen because it is required for exoskeleton formation (Oliver and Meechan, 1993; Warburg et al., 1984).

Based on the results of experiment 1, a density of 6 animals per box was chosen for this experiment. For each diet, 7 replicates were established in a total of 126 individuals with an initial average weight of $10.2 \mathrm{mg}$. The experiment was conducted at a constant temperature of $22^{\circ} \mathrm{C}\left( \pm 1^{\circ} \mathrm{C}\right)$ under a 16-h light:8-h dark regime.

Isopods were weighed every 2 weeks, for 10 (for alder leaves and mixture 1) or 11 weeks (for mixture 2). Food and water were supplied, and the initial isopod density was maintained, as in experiment 1. Parameters measured in this experiment were also the same as in experiment 1.

\section{Data Analysis}

In both experiments growth is measured both as growth rate (weight gain/week) and as percentage of weight gain [(increase in biomass $\times 100$ )/final weight]. Growth rates were calculated using a linear regression (best fit to the data) considering the average weight at each box, not taking into 
account new additions that week. To compare the different growth rates (slopes) a covariance analysis was used in experiment 1 (first generation, three slopes) and experiment 2 (three diets, three slopes); the growth rates of the second generation in experiment 1 (two slopes) were compared with a $t$ test (Zar, 1996).

Percentage weight gain values were compared with an ANOVA (first generation in experiments 1 and 2) or with a $t$ test (second generation in experiment 1). Prior to analysis, data were transformed in $A \sin (x)$ to achieve normality and homogeneity of variances; these assumptions were verified by a Kolmogorov-Sminov and Bartlett or $F$ test, respectively (Zar, 1996).

Reproduction data were compared using nonparametric tests, since normality or homoscedasticity was not achieved even after data transformation. Data resulting from the first generation of experiment 1 and from experiment 2 were compared with a Kruskal-Wallis test and data resulting from the second generation of experiment 1 were compared with a Mann-Whitney test.

\section{RESULTS}

\section{Density Experiment (Experiment 1)}

In the first generation, growth rate was significantly different at the three densities studied $(F=22.76, P<0.05)$ (Table 2). Analyzing the second generation, growth rate was also higher at the lowest density considered (6 animals/box with $1.5 \mathrm{mg}$ /week) when compared with the $1.2 \mathrm{mg}$ /week obtained with 12 animals/box $(t=6.42, P<0.05)$. Percentage weight gain was also higher at the lowest density $(6$ animals/box) in both generations (Fig. 1); in this case, however, no significant differences were obtained $(F=3.14$, $P>0.05$ and $t=0.38, P>0.05$, respectively).

Reproductive results indicated a higher reproductive output at the lowest density in both generations (Fig. 2). In the

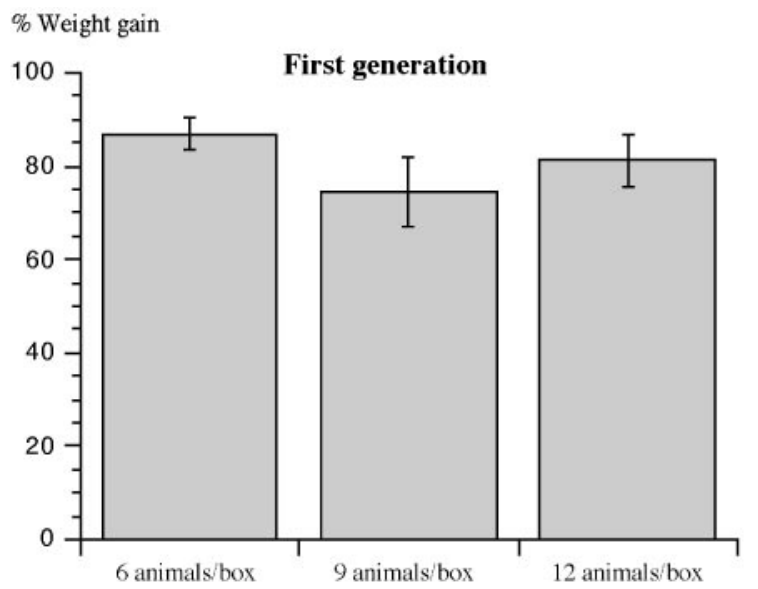

TABLE 2 Growth Rates of $\boldsymbol{P}$. dilatatus in Both Experiments

\begin{tabular}{lccc}
\hline Expt 1 (1 $1^{\text {st }}$ generation $)$ & 6 animals/box & 9 animals/box & 12 animals/box \\
\hline GR (mg/week) & 3.0 & 2.2 & 2.4 \\
$R^{2}$ & 0.81 & 0.77 & 0.65 \\
Expt 2 & Alder leaves & Mixture 1 & Mixture 2 \\
\hline GR (mg/week) & 3.0 & 3.2 & 1.2 \\
$R^{2}$ & 0.80 & 0.84 & 0.84 \\
\hline
\end{tabular}

first generation 38 pregnant females produced an average of 23.4 juveniles/female; in the second generation the average number of juveniles/female was 21.5 at 6 animals/box versus 16.8 at 12 animals/box. However, in both cases, nonparametric tests did not reveal any statistical difference among treatments, probably due to the great variability observed in the results.

In both generations more isopods were found dead at the highest density.

\section{Food Quality Experiment (Experiment 2)}

The analysis of growth curves for the three diets tested indicates that growth rate was higher in isopods fed mixture 1 (Table 2). There was a significant difference among the three types of diet studied $(F=3.77, P<0.05)$. However, the value for mixture 1 is similar to the value obtained with alder leaves, both being higher than the value for mixture 2 . These differences may be explained by the composition of the various food types (Table 1), especially nitrogen content. In this context alder leaves can be considered a highquality food. This is confirmed by the weight gain results, which reveal a high percentage of weight gain in isopods fed

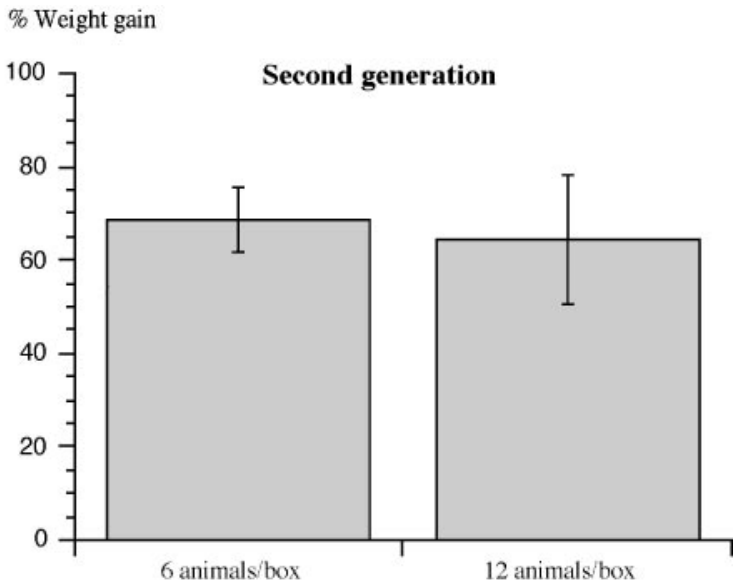

FIG. 1. Percentage weight gain in P. dilatatus (average \pm SD) at the three densities analyzed (first and second generations) for periods of 10 and 18 weeks respectively. 

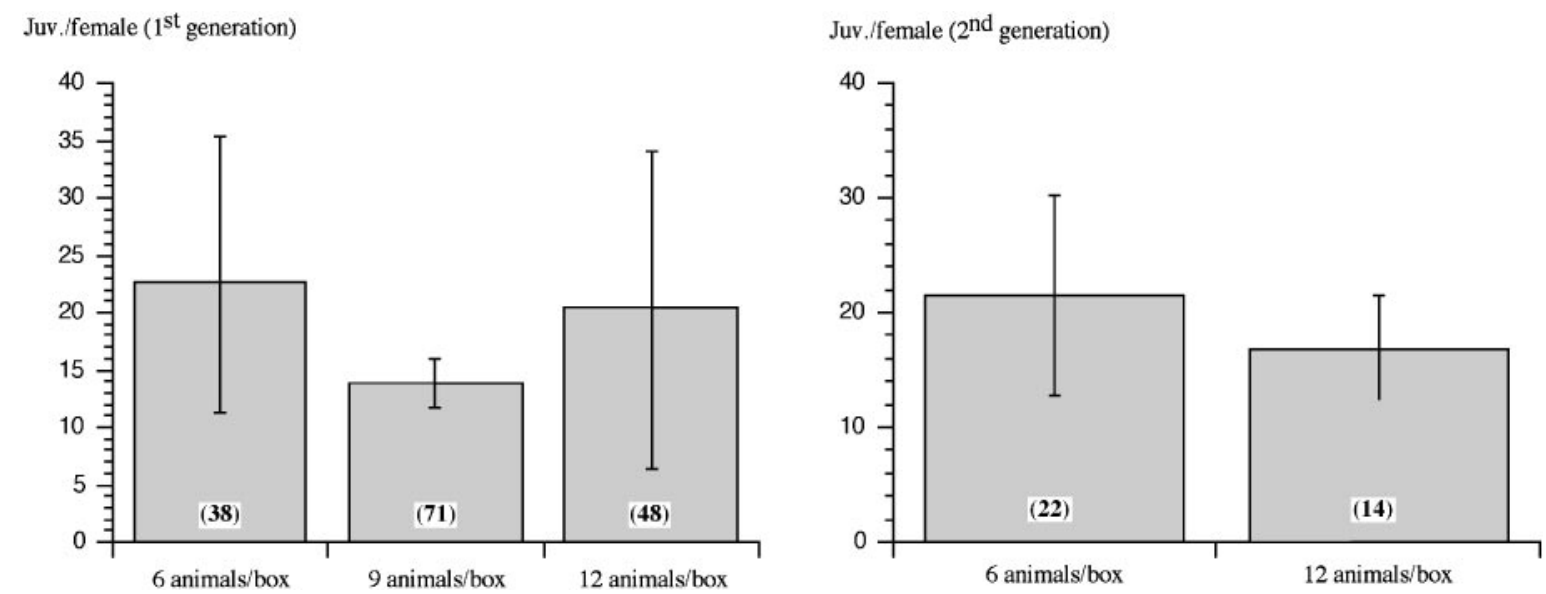

FIG. 2. Reproductive output of $P$. dilatatus (average number of juveniles/female $\pm \mathrm{SD}$ ) at the three densities analyzed (first and second generations) for periods of 10 and 18 weeks, respectively. Figures inside columns indicate numbers of pregnant females.

this diet (Fig. 3). Data analysis revealed statistical differences in this parameter for the three types of food $(F=$ 50.97; $P \ll 0.01$ ).

Reproduction results (Fig. 4) were also favorable to alder leaves, with a larger number of juveniles produced per female when compared with the other diets $(H=14.31$, $P<0.05$ ). The small numbers of juveniles obtained with the two mixtures could be explained by several factors: (1) the type of food itself; (2) the fact that females start to breed earlier in their life cycle (with a weight of $17 \mathrm{mg}$, in contrast to the weight of $22 \mathrm{mg}$ for females of alder leaf cultures); and (3) the experiments with mixtures 1 and 2 were performed in autumn and winter, less favorable periods for reproduction (Mocquard et al., 1976).

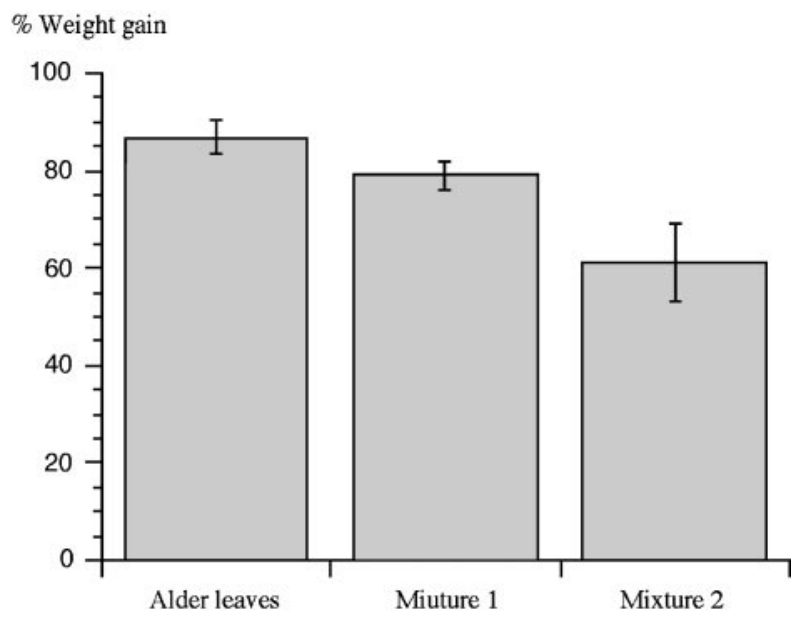

FIG. 3. Percentage weight gain in $P$. dilatatus (average \pm SD) on the three diets tested for periods of 10 weeks (alder leaves and mixture 1 ) and 11 weeks (mixture 2).
Of these three factors, the first two are, undoubtedly, the most important. Food quality may influence reproductive output by controlling both growth and time of first pregnancy and the amount and type of energy reserves allocated to reproduction. With respect to the third factor, animals were maintained in artificial conditions, hence the "season effect" was diminished.

To summarize the results obtained from both experiments and also to demonstrate the advantages of using the appropriate diet and culture density, two simulation diagrams are presented in Fig. 5. Diagram A presents the number of juveniles produced when using cultures at the three densities studied; starting with 100 animals and taking

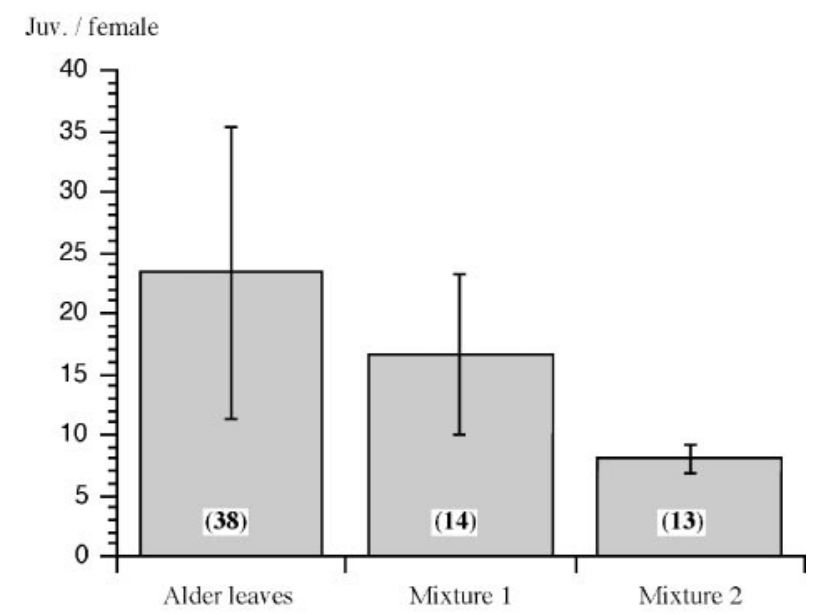

FIG. 4. Reproductive output of $P$. dilatatus (average number of juveniles/female $\pm \mathrm{SD}$ ) on the three diets tested for periods of 10 weeks (alder leaves and mixture 1) and 11 weeks (mixture 2). Figures inside columns indicate numbers of pregnant females. 

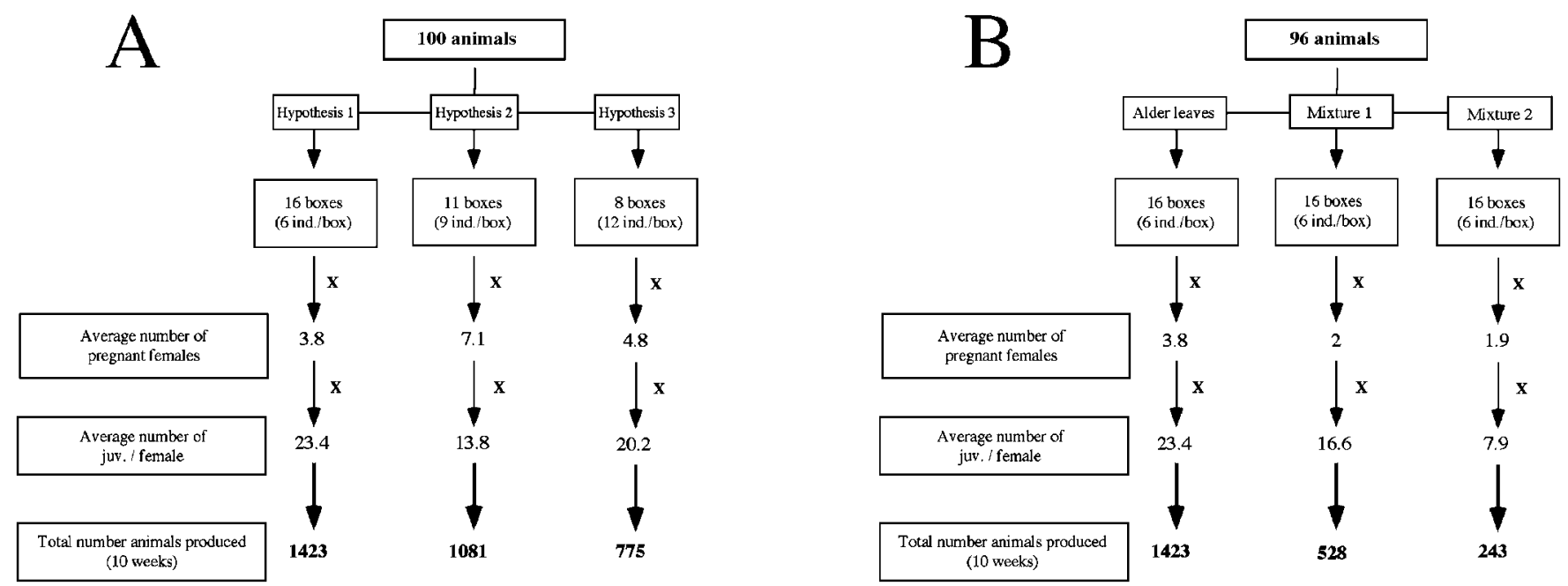

FIG. 5. Simulation diagrams indicating the number of juveniles produced using the number of pregnant females and the reproductive output obtained in both experiments: (A) results using three densities, (B) results using three diets.

into account the values obtained for the measured parameters (pregnant females, juveniles/female), it is possible to see that the number of juveniles produced is higher when the density of 6 animals/box is used.

In diagram $B$ the influence of food type on juvenile production is analyzed using the same principles, but this time always considering a density of 6 animals/box. It is clear that alder leaves induce the best output in terms of juveniles produced when compared with the other diets, reinforcing the quality of this diet.

\section{DISCUSSION}

The primary aim of any culture system to be used in ecological or ecotoxicological tests is the reliable production of large numbers of animals (of both sexes and different age classes) of a consistent quality. The use of cultured animals should also ensure the decrease in the natural variability inherent to the several measured test parameters (ranging from weight to enzymatic baseline values) to facilitate data interpretation. With the use of isopods in laboratory experiments being more generalized, particular attention to culture conditions is needed.

The results obtained in this study clearly indicate the importance of animal density and diet in the maintenance of good laboratory cultures of soil isopods. The first factor (number of individuals per box) is very important since low densities (6 individuals per box) ensure both high growth rates and high reproductive output. On the other hand, mortality rate is low at this density, and a reduced number of animals are used to maintain the initial conditions. These results are in accordance with Hassall (1983) and Ganter (1984) who provided sound evidence that density affects, directly or indirectly, the size of isopod populations. Regarding the size of culture containers, Hornung et al. (1998) mentioned sizes ranging from $4.5 \times 7.5 \mathrm{~cm}$ to $30 \times 20 \times 20 \mathrm{~cm}$; however, these authors never referred to the number of animals inside the boxes. From current results, the use of large culturing containers $(30 \times 20 \mathrm{~cm})$ with 30 animals per container is suggested; in this way the best results for growth, reproduction, and mortality will be achieved.

Perhaps more relevant than animal density, the diet offered plays a key role in the development of a laboratory culture. A proper diet should offer a variety of nutrients and enough energy for growth and reproduction processes, allowing the production of animals in good physiological condition. In this study it was clear that a nitrogen-rich diet induced the best performance of the isopods; mixture 1 produced good isopod growth rate, but alder leaves induced the best reproductive performance.

Farkas et al. (1996) found that growth rates of $P$. scaber were much higher when fed a special complex diet $(50 \%$ ground Acer sp. leaves, $10 \%$ potato powder, and $40 \%$ commercial rabbit food) than when fed only ground Acer sp. leaves. Hornung et al. (1998) obtained similar results with $P$. scaber fed leaves of different tree species (Acer pseudoplatanus, Fagus sylvaticus, Populus alba, Quercus petraea), carrot, and standard rabbit food. These authors found that when only leaves were used, weight increase of isopods was very low because of the low nutritive value of the leaves. This is the major factor explaining the differences observed between these two studies and the results obtained here. It is known that alder leaves are soft and have a high nitrogen content ( $=$ high nutritive value) and even if they are not well conditioned, they can be considered an optimal food for detritus-feeding animals (Sousa et al., 1998). 
Regarding reproduction, food quality may also play an important role in regulating breeding success. Since the number of offspring per female is correlated with the size of females (Zimmer and Topp, 1997), reproductive success appears to be influenced by the nutritional status of the available resources. Some authors (Rushton and Hassall, 1987; Merriam, 1971) have pointed out that a shortage of high-quality food can extend the pre-reproductive period, with consequent deleterious effects to the population. As for growth rate, the nitrogen-rich diet gave the best results. In fact, despite the importance of other nutrients for the development of isopods, nitrogen content seems to be the key factor in establishing the quality of a diet. Together with nutrients, leaf hardness and leaf defenses (e.g., phenolic content) are also important factors in determining food palatability to isopods (Van Wensem et al., 1993).

So, it is advisable to use, as a routine diet, a soft nitrogenrich leaf type, complemented, from time to time, with pieces of carrot or potato to increase the content of some nutrients (e.g., phosphorus). The use of single leaves has the advantage of causing less moldiness in the test boxes, decreasing the workload in maintaining the cultures. When those leaf types are not available, or it is not possible to use well-conditioned leaves, a diet similar in composition to mixture 1 may be used.

\section{CONCLUSIONS}

The results presented in this study confirm the importance of culture conditions when trying to obtain the desired number of animals in optimal physiological condition. In the case of isopods the correct animal density and diet should be regarded as key parameters for the success of a laboratory culture. Besides these parameters, the maintenance of a culture should involve periodic checking of the physiological condition of the individuals (by measuring energy reserves for several individuals) and also the regular evaluation of parameters such as growth and food consumption. Based on experience it is proposed that performing this culture check twice a year is ideal.

\section{ACKNOWLEDGMENT}

This research was supported by Fundação para a Ciência e Tecnologia (Project PBIC/C/AGR/2305/95).

\section{REFERENCES}

Bayley, M. (1995). Prolonged effects of the insecticide dimethoate on locomotor behavior in the woodlouse Porcellio scaber Latr. (Isopoda). Ecotoxicology 4, 74-90.

Brody, M. S., and Lawlor, L. R. (1984). Adaptative variation in offspring size in the terrestrial isopod, Armadillidium vulgare. Oecologia 61, 55-59.

Carefoot, T. H. (1984). Nutrition and growth of Ligia pallasii. Symp. Zool. Soc. London 53, 455-467.
Donker, M. H. (1992). Energy reserves and distribution of metals in populations of the isopod Porcellio scaber from metal contaminated sites. Funct. Ecol. 6, 445-454.

Donker, M. H., Zonneveld, C., and Van Straalen, N. M. (1993). Early reproduction and increased reproductive allocation in metal-adapted populations of the terrestrial isopod Porcellio scaber. Oecologia 96, 316-323.

Drobne, D. (1997). Terrestrial isopods: A good choice for toxicity testing of pollutants in the terrestrial environment. Environ. Toxicol. Chem. 16(6), 1159-1164.

Farkas, S., Hornung, E., and Fisher, E. (1996). Toxicity of copper to Porcellio scaber Latr. (Isopoda) under different nutritional status. Bull. Environ. Contam. Toxicol. 57, 582-588.

Ganter, P. F. (1984). The effects of crowding on terrestrial isopods. Ecology 65, 438-445.

Hassall, M. (1983). Population metabolism of the terrestrial isopod Philoscia muscorum in a dune grassland ecosystem. Oikos 41, 17-26.

Hassall, M., and Dangerfield, J. M. (1997). The population dynamics of a woodlouse, Armadillidium vulgare: An example of biotic compensatory mechanisms amongst terrestrial macrodecomposers? Pedobiologia 41, 342-360.

Hassall, M., and Rushton, S. P. (1984). Feeding behavior of terrestrial isopods in relation to plant defences and microbial activity. Symp. Zool. Soc. London 53, 487-505.

Hopkin, S. P. (1991). A Key to the Woodlice of Britain and Ireland. AIDGAP, London.

Hopkin, S. P., and Martin, M. H. (1982). The distribution of zinc, cadmium, lead, and copper within the woodlouse Oniscus asellus (Crustacea, Isopoda). Oecologia 54, 227-232.

Hornung, E., Farkas, S., and Fisher, E. (1998). Tests on the isopod Porcellio scaber. In Handbook of Soil Invertebrate Toxicity Tests (H. Løkke and C. A. M. van Gestel, Eds.), pp. 207-226. Wiley, Chichester.

Léon, C. D., and Van Gestel, C. A. M. (1994). Selection of a Set of Laboratory Ecotoxicity Tests for the Effect Assessment of Chemicals in Terrestrial Ecosystems, Discussion Paper. Free Univ. Press.

Merriam, G. (1971). Sensitivity of terrestrial isopod populations (Armadillidium) to food quality differences. Can. J. Zool. 49, 667-674.

Mocquard, J. P., Besse, G., Juchault, P., Legrand, J. J., Maissiat, J., Martin, G., and Picaud, J. L. (1976). Durée de la période de reproduction chez les femelles de l'oniscoide Porcellio dilatatus suivant les conditions d'élevage: temperature, photopériode et groupement. Vie Milieu. 26(1-C), 51-76.

Oliver, P. G., and Meechan, C. J. (1993). Woodlice (Synopses of the British Fauna), Doriskermark Editor, No. 4. Field Studies.

Sousa, J. P., Vingada, J. V., Loureiro, S., Gama, M. M., and Soares, A. M. V. M. (1998). Effects of introduced exotic tree species on growth, consumption and assimilation rates of the soil detritivore Porcellio dilatatus (Crustacea: Isopoda). Appl. Soil Ecol. 9, 399-403.

Römbke, J., Bauer, C., and Marschner, A. (1996). Hazard assessment of chemicals in soil: Proposed ecotoxicological test strategy. Environ Sci. Pollut. Res. 3, 78-82.

Rushton, S. P., and Hassall, M. (1983a). The effects of food quality on the life history parameters of the terrestrial isopod (Armadillidium vulgare (Latreille)). Oecologia 57, 257-261.

Rushton, S. P., and Hassall, M. (1983b). Food and feeding rates of the terrestrial isopod Armadillidium vulgare (Latreille). Oecologia 57, 415-419.

Rushton, S. P., and Hassall, M. (1987). Effects of food quality on isopod population dynamics. Funct. Ecol. 1, 359-367.

Van Straalen, N. M., and Verweij, R. A. (1991). Effects of benzo(a)pyrene on food assimilation and growth efficiency in Porcellio scaber. Bull. Environ. Contam. Toxicol. 46, 134-140. 
Van Wensem, J. (1989). A terrestrial micro-ecosystem for measuring effects of pollutants on isopod-mediated litter decomposition. Hidrobiologia 188/189, 507-516.

Van Wensem, J., Verhoef, H. A., and Van Straalen, N. M. (1993). Litter degradation stage as a prime factor for isopod interaction with mineralization processes. Soil Biol. Biochem. 9, 1175-1183.

Vandel, A. (1962). Isopodes terrestres. In Faune de France (P. leChevalier, Ed.), pp. 417-931. Paris.

Warburg, M. R. (1995). Growth and reproduction in a rare desert isopod: Porcellio barroisi (Oniscidea; Porcellionidae) from the Central Negev Mountains. J. Arid Environ. 31, 199-204.
Warburg, M. R., Linsenmair, K. E., and Bercovitz, K. (1984). The effect of climate on the distribution and abundance of isopods. Symp. Zool. Soc. London 53, 339-367.

Wieser, W., and Oberhauser, C. (1984). Ammonia production and oxygen consumption during the life cycle of Porcellio scaber (Isopoda, crustacea). Pedobiologia 26, 415-419.

Zar, J. H. (1996). Biostatistical Analysis, 3rd ed., Prentice-Hall, London.

Zimmer, M., and Topp, W. (1997). Does leaf litter quality influence population parameters of the common woodlouse, Porcellio scaber (Crustacea: Isopoda)? Biol. Fertil. Soils 24, 435-441. 